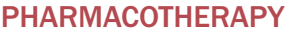

\section{Benefit of testosterone replacement therapy on CVD and mortality}

The use of testosterone replacement therapy (TRT) in men is on the rise, but its effect on mortality and cardiovascular disease (CVD) remains uncertain. Sharma and colleagues evaluated the relationship between TRT normalization of total testosterone (TT), and all-cause mortality and cardiovascular events (defined as risk of myocardial infarction [MI] and stroke). A total of 83,010 male veterans with documented low TT levels were included in this retrospective study. Participants were divided into three groups: group 1 , TRT with eventual normalization of TT levels $(n=43,931)$; group 2 , TRT without normalization of TT levels $(n=25,701)$; and group 3 , those who did not receive TRT $(n=13,378)$. Using a propensity score-weighted Cox proportional hazard model, all-cause mortality (HR 0.44, 95\% CI 0.42-0.46), risk of MI (HR 0.76, 95\% CI 0.63-0.93), and stroke (HR 0.64, 95\% CI 0.43-0.96) were found to be significantly lower in patients receiving TRT who had normalization of TT levels than in patients who did not receive TRT. Furthermore, all-cause mortality, risk of MI, and stroke were lower in group 1 than in group 2, leading the study investigators to suggest that "patients who failed to achieve the therapeutic range after TRT did not see a reduction in MI or stroke". They acknowledge that while normalization of TT levels after TRT was beneficial in lowering CVD risk and mortality, "the mechanisms for these effects remain speculative" and "future, adequately powered, prospective, well-designed trials with a long-term follow-up will be needed".

\section{Karina Huynh}

Original article Sharma, R. et al. Normalization of testosterone level is associated with reduced incidence of myocardial infarction and mortality in men. Eur. Heart J. doi:10.1093/eurheartj/ehv346 